\section{Excretion of Catechol after Ingestion of Quinic and Shikimic Acids}

ThE detection of catechol in acid-hydrolysed normal human urine and cow's urine was reported recently by von Euler and Lishajko ${ }^{1}$. The origin of the catechol was not known. The excretion of hippuric acid in the urine of man after oral ingestion of calcium quinate was reported by Lautemann in $1863^{2}$. It occurred to us that if an aromatic ring can be formed in the animal body from quinic acid ( $1: 3: 4: 5$-tetrahydroxycyclohexane carboxylic acid), then urinary catechol might have a similar origin. Further, an exogenous (dietary) precursor is implicated since we are unable to detect catechol in the urine of rats fed a highly purified diet. Quinic and shikimic acids are known to be present in edible plants.

Adult albino rats were fed a purified diet (sucrose, casein, salts, oil, vitamins) and given quinic acid by stomach tube (100 mgm./rat). A 48-hr. urine sample was collected and subjected to two-dimensional paper chromatographic analysis. The lower phase of a chloroform/acetic acid/water (2:1:1) mixture was used in the first direction followed by 20 per cent potassium chloride in the second direction. Each developed chromatogram was air-dried and carefully examined under short-wave ultra-violet light and then sprayed with diazotized sulphanilic acid followed by 20 per cent sodium carbonate. Thus the positive identification of a urinary metabolite was based on its $R_{F}$ values in two dimensions, its behaviour under ultra-violet light and its colour with diazotized sulphanilic acid-sodium carbonate.

In our chromatographic system ${ }^{3}$ hippuric acid is located at $R_{F} 0.60 \times 0.87$, gives no colour with the diazotized sulphanilic acid reagent and absorbs ultra-violet light. The only notable deviation from normal in the case of the ether extract of the acidified urine of rats given quinic acid by stomach tube was a marked increase in the size of the area corresponding to hippuric acid. Catechol was not detected. Similarly, catechol was not detected after acid hydrolysis (2 $N$ hydrochloric acid reflux, 30 min.) of the ether extract, suggesting that this fraction does not contain an ether-soluble conjugate of catechol. Finally, the ether-extracted urine was acid-hydrolysed and reextracted with ether. When this ether extract was examined chromatographically, catechol was readily detected at $R_{F} 0.50 \times 0.74$. Catechol absorbs ultra-violet light and when sprayed with the diazotized sulphanilic acid reagent undergoes a very characteristic colour change from red to green to blue-black. Another characteristic of catechol is its tendency to darken on an unsprayed chromatogram, presumably due to spontaneous oxidation.

When shikimic acid was administered to rats by stomach tube $(100 \mathrm{mgm}$./rat) the same results were obtained as described above for quinic acid. Thus catechol was clearly present on the chromatogram of the acid-hydrolysed ether-extracted urine. In addition, an unidentified metabolite of both quinic and shikimic acids having the same chromatographic characteristics as vanillic acid was detected in the urine after hydrolysis.

Rats were also fed quinic acid mixed in the purified. diet at a level of 3 per cent. Catechol was readily detected free (unconjugated) as well as conjugated in the urine. According to Garton and Williams ${ }^{4}$, catechol is formed and excreted principally as a mono- glucuronide or monosulphate derivative in the urine of rabbits receiving benzene by stomach tube.

It is difficult to estimate the amount of catechol excreted in urine, due to its instability, especially when subjected to acid hydrolysis. A minimal amount of $2 \mathrm{mgm}$. of catechol was excreted during a period of two days by two rats ingesting a total of $1.5 \mathrm{gm}$. of quinic acid in the diet.

Whether aromatization of quinic and shikimic acids to form catechol is dependent on intestinal micro-organisms is not known. Ayengar et al. ${ }^{5}$ reported that "extracts of rabbit-liver acetone powder catalysed the formation of catechol from 3,5-cyclohexadiene-1,2-diol", but not from D-quinic acid. Mitoma et al. isolated an enzyme system from guinea pig liver which was able to aromatize cyclo. hexanecarboxylic acid ${ }^{6}$.

From these results it is tempting to conclude that the catechol in human and cow urine may be formed from quinic acid and related substances in the diet.
A. N. Воотн
D. J. Robbins
M. S. MASRI
F. DeEds

Western Regional Research Laboratory,

Agricultural Research Service,

U.S. Department of Agriculture,

Albany,

California.

${ }^{1}$ Euler, U. S. v., and Lishajko, F., Nature, 183, 1123 (1959).

${ }^{2}$ Lautemann, E., Liebigs Ann., 125, 9 (1863).

Booth, A. N., Emerson, O. H., Jones, F. T., and DeEds, F., J. Biol. Chem., 229, 51 (1957).

${ }^{4}$ Garton, G. A., and Williams, R. T., Biochem. J., 43, 206 (1948).

${ }^{5}$ Ayengar, P. K., Hayaishi, O., Nakajima, M., and Tomida, I., Biochim. Biophys, Acta, 33, 111 (1959).

${ }^{6}$ Mitoma, C., Posner, H. S., and Leonard, F., Biochim. Biophys. Acta, 2ri', 156 (1958).

\section{Staining for lodine in Chromatograms of Human Plasma : an Artefact due to Thiourea or Thiouracils}

UNTIL the report by Werner and Block ${ }^{1}$, it was generally thought that at most only very small quantities of iodinated tyrosines are present in normal human serum. This conclusion was based on ${ }^{231} \mathrm{I}$. chromatographic studies using plasma extracts from subjects given radioiodine. Werner and Block report, however, that when the iodine distribution in normal human serum is analysed chemically by the ceric-arsenite reaction ${ }^{2}$, approximately half the circulating iodine is present in the form of monoiodotyrosine or di-iodotyrosine, although the radioactivity in the same chromatogram is distributed according to the results of other workers. Hithorto these results have been very difficult to understand, and they are inconsistent with the rapid deiodination seen when iodotyrosines are injected ${ }^{3}$. We have recently confirmed Werner and Block's results indicating a discrepancy botween the distribution of stable and radioactive iodine, when using very similar methods to estimate the distribution of iodinated amino-acids in human serum. Our extraction and chromatographic techniques differed from those of Mandl and Block ${ }^{4}$ only in that the acidified serum was extracted three times with butanol and that the chromatogram was unidimensional and run in acidbutanol / $2 N$ acetic acid $(1: 1)$. We find that when 